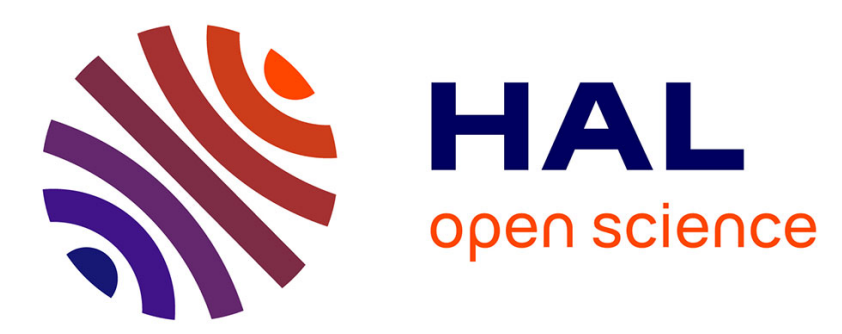

\title{
The representation of natural risk statistics on the space of bounded sequences by coherent risk statistics
}

\author{
Elouali Aailal, A. Hassouni
}

\section{To cite this version:}

Elouali Aailal, A. Hassouni. The representation of natural risk statistics on the space of bounded sequences by coherent risk statistics. International Journal of Mathematical Analysis, 2014, 8, pp.2439 - 2443. 10.12988/ijma.2014.48285. hal-03015644

\section{HAL Id: hal-03015644 https://hal.science/hal-03015644}

Submitted on 20 Nov 2020

HAL is a multi-disciplinary open access archive for the deposit and dissemination of scientific research documents, whether they are published or not. The documents may come from teaching and research institutions in France or abroad, or from public or private research centers.
L'archive ouverte pluridisciplinaire HAL, est destinée au dépôt et à la diffusion de documents scientifiques de niveau recherche, publiés ou non, émanant des établissements d'enseignement et de recherche français ou étrangers, des laboratoires publics ou privés. 
International Journal of Mathematical Analysis

Vol. 8, 2014, no. 49, $2439-2443$

HIKARI Ltd, www.m-hikari.com

http://dx.doi.org/10.12988/ijma.2014.48285

\title{
The Representation of Natural Risk Statistics on the Space of Bounded Sequences by Coherent Risk Statistics
}

\author{
E. Aailal \\ Department of Mathematics and Informatics, Science Faculty \\ University Mohammed V-Agdal, B.P.1014, Rue Ibn Batouta, Rabat, Morocco

\begin{abstract}
A. Hassouni
Department of Mathematics and Informatics, Science Faculty University Mohammed V-Agdal, B.P.1014, Rue Ibn Batouta, Rabat, Morocco

Copyright (c) 2014 E. Aailal and A. Hassouni. This is an open access article distributed under the Creative Commons Attribution License, which permits unrestricted use, distribution, and reproduction in any medium, provided the original work is properly cited.
\end{abstract}

\begin{abstract}
In this paper, we present a representation theorem of natural risk statistics on the space of infinite and bounded real sequences via coherent risk statistics.
\end{abstract}

Keywords: natural risk statistics, coherent risk statistics, convex conjugate, representation theorem

\section{Introduction}

Heyde et al. [4].(2006) proposed the notion of natural risk statistics on the vector space of finite samples, instead of random variables as in the classical approach, first introduced in [2] by Artzner et al.(1999). By weakening the subadditivity axiom, these measures of risk are consistent with industrial practice and have some interesting features which make them consistent with 
scenario analysis used in practice. Assa et al. [3].(2010) presented a generalization of natural risk statistics to the space of infinite and bounded sequences $l^{\infty}$, motivated by their work, we introduce an alternative proof of the main result in [3] which states that a weak-star lower semicontinuous risk statistics on $l^{\infty}$ is natural if and only if, it can be represented as a supremum over a family of convex combinations of order statistics.

\section{Preliminary Notes}

In this section we recall some definitions and results regarding coherent and natural risk statistics.

Definition 2.1 ([3,5]) Let $f: E \rightarrow \mathbb{R} \cup\{-\infty,+\infty\}$, where $E$ is a metric space. We say that $f$ is lower semicontinuous, if $f(x) \leq \liminf _{y \rightarrow x} f(y) \forall x \in E$.

Definition 2.2 ([5]) Let $f: E \rightarrow \mathbb{R} \cup\{\infty\}$, where $E$ is a locally convex topological vector space, the Fenchel-Legendre transform of $f$ is the function $f^{*}(l)=\sup _{x \in E}(\langle l, x\rangle-f(x)), l \in E^{\prime}$ where $E^{\prime}$ is the topological dual of $E$.

Theorem 2.3 ([6]) If $f: l^{\infty} \rightarrow \mathbb{R} \cup\{\infty\}$ is a lower semicontinuous, convex function, then $f(X)=\sup _{w \in W}\left(\langle w, X\rangle-f^{*}(w)\right) \forall X \in l^{\infty}$, where $W=\operatorname{dom}\left(f^{*}\right)$ (The domain of $f^{*}$ ) is a closed convex set. Moreover:

(a) $f$ is monotone if and only if $w \geq 0 \forall w \in W$;

(b) $f$ is translation invariant if and only if $\sum_{i=1}^{\infty} w_{i}=1 \forall w \in W$;

(c) $f$ is positively homogenous if and only if $f^{*}(w)=0 \forall w \in W$.

Definition 2.4 ([2,4]) A function $\rho: l^{\infty} \rightarrow \mathbb{R}$ is a coherent risk statistics, if:

1- $\rho$ is invariant by translation and positively homogeneous:

$\rho(\lambda X+c \mathbf{1})=\rho\left(\lambda x_{1}+c, \lambda x_{2}+c, \lambda x_{3}+c, \ldots\right)=\lambda \rho\left(x_{1}, x_{2}, x_{3}, \ldots\right)+c=\lambda \rho(X)+c$ $\forall \lambda \geq 0$ and $\forall c \in \mathbb{R}$, where $\mathbf{1}=(1,1,1, \ldots) \in l^{\infty}$;

2- $\rho$ is monotone: If $X \leq Y$ (i.e., $\left.x_{1} \leq y_{1}, x_{2} \leq y_{2}, x_{3} \leq y_{3}, \ldots\right)$, then $\rho(X)=\rho\left(x_{1}, x_{2}, x_{3}, \ldots\right) \leq \rho(Y)=\rho\left(y_{1}, y_{2}, y_{3}, \ldots\right)$;

3- $\rho$ is subadditive: $\forall X, Y \in l^{\infty}$

$\rho(X+Y)=\rho\left(x_{1}+y_{1}, x_{2}+y_{2}, \ldots\right) \leq \rho\left(x_{1}, x_{2}, \ldots\right)+\rho\left(y_{1}, y_{2}, \ldots\right)=\rho(X)+\rho(Y)$.

The next theorem is the well-known representation theorem in [2] of coherent risk measures, stated here in $l^{\infty}$, where $\forall X, Y \in l^{\infty}\langle X, Y\rangle=\sum_{i=1}^{\infty} x_{i} y_{i}$.

Theorem 2.5 ([2]) Let $\rho$ be a function on $l^{\infty}$. The function $\rho$ is a coherent risk statistics if and only if, $\rho(X)=\sup _{w \in W}\langle w, X\rangle \forall X \in l^{\infty}$ where we have $W \subseteq\left\{w \in l^{\infty}: w \geq 0, \sum_{i=1}^{\infty} w_{i}=1\right\}$ is a closed, convex set of $l^{1}$. 
Now, we present the definition of a natural risk statistics on $l^{\infty}$, as it was extended in [3], from $\mathbb{R}^{\mathrm{n}}$ to $l^{\infty}$.

Definition 2.6 ([3]) A function $\rho: l^{\infty} \rightarrow \mathbb{R}$ is a natural risk statistics, if: 1- $\rho$ is invariant by translation and positively homogeneous:

$\rho(\lambda X+c \mathbf{1})=\rho\left(\lambda x_{1}+c, \lambda x_{2}+c, \lambda x_{3}+c, \ldots\right)=\lambda \rho\left(x_{1}, x_{2}, x_{3}, \ldots\right)+c=\lambda \rho(X)+c$ $\forall \lambda \geq 0$ and $\forall c \in \mathbb{R}$, where $\mathbf{1}=(1,1,1, \ldots) \in l^{\infty}$;

2- $\rho$ is monotone: If $X \leq Y$ (i.e., $\left.x_{1} \leq y_{1}, x_{2} \leq y_{2}, x_{3} \leq y_{3}, \ldots\right)$, then $\rho(X)=\rho\left(x_{1}, x_{2}, x_{3}, \ldots\right) \leq \rho(Y)=\rho\left(y_{1}, y_{2}, y_{3}, \ldots\right)$;

3- $\rho$ is comonotone subadditive:

If $X$ and $Y$ are comonotonic (i.e., $\left(x_{i}-x_{j}\right)\left(y_{i}-y_{j}\right) \geq 0$ for any $\left.j \neq i\right)$, then $\rho(X+Y)=\rho\left(x_{1}+y_{1}, x_{2}+y_{2}, \ldots\right) \leq \rho\left(x_{1}, x_{2}, \ldots\right)+\rho\left(y_{1}, y_{2}, \ldots\right)=\rho(X)+\rho(Y)$; 4- $\rho$ is invariant under permutation: for any permutation $\pi=\left(i_{1}, i_{2}, i_{3}, \ldots\right)$ of $\mathbb{N}^{*}$, we have $\rho(X)=\rho\left(x_{1}, x_{2}, x_{3}, \ldots\right)=\rho\left(x_{i_{1}}, x_{i_{2}}, x_{i_{3}}, \ldots\right)=\rho(\pi(X))$.

\section{Main Results}

We define the set $D=\left\{X \in l^{\infty}: x_{1} \geq x_{2} \geq x_{3} \geq, \ldots, \geq x_{n} \geq, \ldots\right\}$. Let $D^{\circ}$ denote the interior of $\mathrm{D}$ and $\operatorname{Fr}(\mathrm{D})$ its frontier or boundary, $\chi_{D}$ is the indicator function of D, i.e., $\chi_{D}(X)=\left\{\begin{array}{cc}0 & , X \in D \\ +\infty & , \text { o.w. }\end{array}\right.$. We consider for every element $X \in l^{\infty}$ the set $s_{X}=\left\{x_{0}=\lim \sup X\right\} \cup\left\{x_{i}: x_{i} \geq x_{0}\right\}$ with multiplicity $\left(s_{X}\right.$ contains all the copies of the components of $\mathrm{X}$ which are larger than $\left.x_{0}\right)$ and the element $(\Lambda(X))_{i}=\left\{\begin{array}{cc}\max _{i} s_{X} & ,(\Lambda(X))_{i-1}>x_{0} \\ x_{0} & , o . w .\end{array}\right.$ of $l^{\infty}$ where $\max _{i} s_{X}$ denotes the i-th biggest number of $s_{X}$.

Lemma 3.1 Let $\rho: l^{\infty} \rightarrow \mathbb{R}$ be a natural risk statistics and $\hat{\rho}: l^{\infty} \rightarrow \mathbb{R}$ be a coherent risk statistics, we have $\rho(X)=\hat{\rho}(\Lambda(X)) \forall X \in l^{\infty}$ if and only if $\rho(X)=\hat{\rho}(\Lambda(X))=\hat{\rho}(X) \forall X \in D$.

Proof. If this is not true, then $\exists X^{0} \in\left(l^{\infty} \backslash D\right)$ such that $\rho\left(X^{0}\right) \neq \hat{\rho}\left(\Lambda\left(X^{0}\right)\right)$, since $\Lambda\left(X^{0}\right) \in D$, then $\hat{\rho}\left(\Lambda\left(X^{0}\right)\right)=\rho\left(\Lambda\left(X^{0}\right)\right) \neq \rho\left(X^{0}\right)$ which is impossible because $\rho$ is invariant by permutation.

Remark 3.2 A real-valued coherent or natural risk statistics on $l^{\infty}$ is Lipschitz continuous and convex. Hence, it is continuous in the strong topology which is the topology induced by the supremum norm, then it is weak lower semicontinuous, but not necessary weak-star lower semicontinuous on $l^{\infty}$.

Now, we are in a position to state a relationship between coherent risk statistics and natural risk statistics. 
Theorem 3.3 A function $\rho: l^{\infty} \rightarrow \mathbb{R}$ is a natural risk statistics if and only if, there exists a coherent risk statistics $\hat{\rho}: l^{\infty} \rightarrow \mathbb{R}$ such that $\rho(X)=\hat{\rho}(\Lambda(X))$ $\forall X \in l^{\infty}$.

Proof $\bullet$ If $\rho$ is a natural risk statistics, let $\tilde{\rho}(X)=\rho(X)+\chi_{D}(X) \forall X \in l^{\infty}$. The function $\tilde{\rho}$ is:

-Convex: If $\mathrm{X}$ and $\mathrm{Y}$ are both in $\mathrm{D}$, then $\lambda X+(1-\lambda) Y \in D \forall \lambda \in[0,1]$, so $\tilde{\rho}$ is convex because $\rho$ is convex on $\mathrm{D}$. If $X \notin D$ or $Y \notin D$, then $\chi_{D}(X)=+\infty$ or $\chi_{D}(Y)=+\infty$, which means that $\tilde{\rho}(\lambda X+(1-\lambda) Y) \leq \lambda \tilde{\rho}(X)+(1-\lambda) \tilde{\rho}(Y)$ $\forall \lambda \in[0,1]$. hence, $\tilde{\rho}$ is convex on $l^{\infty}$.

-Invariant by translation: If $X \in D$, then $\forall c \in \mathbb{R}$ by translation invariance of $\rho$ we obtain $\tilde{\rho}(X+c \mathbf{1})=\rho(X+c \mathbf{1})=\rho(X)+c=\tilde{\rho}(X)+c$. If $X \notin D$, then $\tilde{\rho}(X+c \mathbf{1})=\tilde{\rho}(X)+c=+\infty$. Thus, $\tilde{\rho}$ is translation invariant.

-Positively homogeneous: $\mathrm{D}$ is a positive cone. If $X \in D$, then $\forall \lambda>0 \lambda X \in D$ and $\chi_{D}(\lambda X)=0$, by the positive homogeneity of $\rho$ we have $\tilde{\rho}(\lambda X)=\rho(\lambda X)=$ $\lambda \rho(X)=\lambda \tilde{\rho}(X)$. If $X \notin D$, then $\tilde{\rho}(\lambda X)=\lambda \tilde{\rho}(X)=+\infty$.

-Considering the theorem 2.3 and the remark 3.2, $\tilde{\rho}$ could be represented as follows: $\tilde{\rho}(X)=\sup _{v \in V^{\prime}}\langle v, X\rangle$ where $V^{\prime}$ is a convex and closed subset of the set $\left\{v \in l^{\infty}: \sum_{i=1}^{\infty} v_{i}=1\right\}$.

-We construct $\hat{\rho}$ as for every element $\mathrm{X}$ of $l^{\infty} \hat{\rho}(X)=\sup _{v \in V}\langle v, X\rangle$ where $V=\left\{X \in l^{\infty}: X \geq 0\right\} \cap V^{\prime}$. The set $\mathrm{V}$ is non empty because $\hat{\rho}(X)<\infty$ $\forall X \in D$. Moreover, by compactness of $\mathrm{V}$ the supremum of $\langle v, X\rangle$ over $\mathrm{V}$ is achieved $\forall X \in l^{\infty}$, then $\hat{\rho}$ is well defined. $\hat{\rho}$ is convex, as a maximum of a collection of convex functions $\sup _{v \in V}\langle v, X\rangle$.

-Now we will show that $\hat{\rho}(X)=\rho(X) \forall X \in D$. If $X \in \operatorname{Fr}(D)$, we consider the sequence $X^{k} \in D^{\circ}$ ) such that $\lim _{k \rightarrow+\infty} X^{k}=X$ in the usual supremum norm of $l^{\infty}$, by the fact that $\rho$ is continuous and that $\mathrm{V}$ is compact, we obtain $\rho(X)=$ $\lim _{k \rightarrow+\infty} \rho\left(X^{k}\right)=\lim _{k \rightarrow+\infty} \sup _{v \in V}\left\langle v, X^{k}\right\rangle=\sup _{v \in V} \lim _{k \rightarrow+\infty}\left\langle v, X^{k}\right\rangle=\sup _{v \in V}\langle v, X\rangle=$ $\hat{\rho}(X)$. If $X=\left(x_{1}, x_{2}, x_{3}, \ldots\right) \in D^{\circ}$, then $Y_{i}=\left(x_{1}, x_{2}, x_{3}, \ldots, x_{i}-\epsilon, \ldots\right) \in D$ $\forall i=1,2,3, \ldots$, for $\epsilon$ small enough. On another side $\tilde{\rho}$ is monotone on $\mathrm{D}$, so $\left\langle v, Y_{i}\right\rangle \leq \tilde{\rho}\left(Y_{i}\right) \leq \tilde{\rho}(X) \forall v \in V^{\prime}$, then $\left\langle v, Y_{i}\right\rangle \leq\langle v, X\rangle$ which means that $v_{i} \geq 0$ $\forall i=1,2,3, \ldots$. Hence $\rho(X)=\tilde{\rho}(X)=\sup _{v \in V}\langle v, X\rangle=\hat{\rho}(X)$. So, $\hat{\rho}(X)=\rho(X)$ $\forall X \in D$, by the lemma 3.1 we obtain $\rho(X)=\hat{\rho}(\Lambda(X)) \forall X \in l^{\infty}$.

- Suppose that $\hat{\rho}$ is a coherent risk statistics. It is clear that $\forall(\lambda, c) \in \mathbb{R}^{+} \times \mathbb{R}$ $\Lambda(\lambda X+c)=\lambda \Lambda(X)+c$, which means that $\Lambda$ is invariant by translation and positively homogeneous, also $\Lambda$ is naturally permutation invariant. if $X \leq Y$ then $\lim \sup X=x_{0} \leq \limsup Y=y_{0}$ and $\max _{i} s_{X} \leq \max _{i} s_{Y} \forall i=1,2,3, \ldots$, so, $(\Lambda(X))_{i} \leq(\Lambda(Y))_{i}$ which means that $\Lambda$ is increasing.

-Now $\hat{\rho}$ as a coherent risk statistics, composed with $\Lambda$, is translation invariant, increasing, positively homogeneous and invariant by permutation. To see that $\rho(X)=\hat{\rho}(\Lambda(X))$ is comonotone subadditive, we have $\forall X \in D \Lambda(X)=X$ 
and $\forall X, Y \in D X+Y \in D$, so, $\rho$ is convex over D. Thus, $\rho$ is comonotone subadditive on $l^{\infty}$. Finally, $\rho(X)=\hat{\rho}(\Lambda(X))$ is a natural risk statistics on $l^{\infty}$.

The next corollary presents the main theorem stated in [3].

Corollary 3.4 Let $\rho$ be a real-valued function on $l^{\infty}$. $\rho$ is a weak-star lower semicontinuous natural risk statistics if and only if, $\rho(X)=\sup _{w \in W}\langle w, \Lambda(X)\rangle$ $\forall X \in l^{\infty}$ where $W \subseteq\left\{w \in l^{\infty}: w \geq 0, \sum_{i=1}^{\infty} w_{i}=1\right\}$ is a closed, convex set of $l^{1}$.

Proof. Combining the theorem 2.5, the theorem 3.3 and the remark 3.2 we obtain the result.

\section{References}

[1] S. Ahmed, D. Filipovic, G. Svindland, A note on natural risk statistics , Operations Research Letters, 36 (2008), 662 - 664.

[2] P. Artzner, F. Delbaen, J.-M. Eber, D. Heath, Coherent measures of risk, Mathematical Finance, 9(3) (1999), 203 - 228.

[3] H. Assa, M. Morales, Risk measures on the space of infinite sequences, Mathematics and Financial Economics, 2(4) (2010), 253 - 275.

[4] C. C. Heyde, S. G. Kou, X. H. Peng, What is a good risk measure: Bridging the gaps between data, coherent risk measures, and insurance risk measures, Working paper, Columbia University, (2006).

[5] R. T. Rockafellar, Conjugate duality and optimization, Philadelphia : Society for Industrial and Applied Mathematics, (1974).

[6] A. Ruszczyński, A. Shapiro, Optimization of convex risk functions, Mathematics of Operations Research, 31 (2006), 433 - 452.

Received: September 20, 2014, Published: October 27, 2014 